\title{
Frontières
}

\section{Lettres pour un aveugle}

\section{Martine Delvaux}

Volume 14, numéro 2, printemps 2002

La mort prononcée

URI : https://id.erudit.org/iderudit/1073980ar

DOI : https://doi.org/10.7202/1073980ar

Aller au sommaire du numéro

Éditeur(s)

Université du Québec à Montréal

ISSN

1180-3479 (imprimé)

1916-0976 (numérique)

Découvrir la revue

Citer ce document

Delvaux, M. (2002). Lettres pour un aveugle. Frontières, 14(2), 87-90.

https://doi.org/10.7202/1073980ar

Ce document est protégé par la loi sur le droit d'auteur. L'utilisation des services d'Érudit (y compris la reproduction) est assujettie à sa politique d'utilisation que vous pouvez consulter en ligne.

https://apropos.erudit.org/fr/usagers/politique-dutilisation/
Cet article est diffusé et préservé par Érudit.

Érudit est un consortium interuniversitaire sans but lucratif composé de l’Université de Montréal, l'Université Laval et l'Université du Québec à Montréal. Il a pour mission la promotion et la valorisation de la recherche. https://www.erudit.org/fr/ 


\section{LETTRES POUR UN AVEUGLE}

\author{
Martine Delvaux, Ph.D., \\ professeure, Département de littérature, UQÀM.
}

Au moment où je commence à penser ce texte, je viens de terminer «Lettres sur un aveugle » de Jacques Derrida, texte qu'il a écrit pour faire suite à un film fait sur lui, D'ailleurs, Derrida (Paris, Galilée, 2000), par Safaa Fathy. Derrida, on le sait, a déjà écrit sur ceux qui ne voient pas (entre autres dans Mémoires d'aveugle), et il le fait ici comme sur lui, celui qui cherche à se voir être vu par celle qui tourne un film à son sujet, qui le prend en images, qui fait un montage d'images dans le but de le cerner. Et si Safaa Fathy a tourné sur lui des images, Derrida, en retour, se tourne vers le film par le biais d'un abécédaire, écrivant quelques lettres, tournant «autour de quelques mots» (Derrida, 2000, p. 77), autour de certains lieux du film.

Au moment où je commence à penser ce texte, mon grand-père vient de mourir après une agonie ponctuée d'injections de morphine. Il avait 95 ans. Au cours des dernières années de sa vie, il avait presque perdu la vue. Il s'en plaignait régulièrement, tentant de me décrire, de me faire voir ce qui lui restait de vision, de point de vue, comment il ne percevait que certains contours, comment les choses demeuraient éternellement floues. Quand je me trouvais devant lui, il me reconnaissait, mais il ne me voyait pas vraiment; il ne voyait qu'autour de moi, il ne voyait que l'autour de qui j'étais. Il me voyait comme à travers un voile; il me voyait voilé(e).

C'est autour de cette image de l'aveugle que je veux me tourner aujourd'hui, aveugle qui, dans la lecture que Derrida fait du film, figure de façon métonymique l'essence même du processus filmique. Derrida s'arrête sur le plan de l'aveugle de Tolède, filmé par Safaa Fathy dans D'ailleurs, Derrida. «Aveugle» est le premier mot sur lequel il s'arrête dans la composition de son abécédaire, et c'est pour lui, pour cet aveugle de Tolède (comme le titre l'indique) qu'il écrit ses lettres, qu'il tourne ses mots. Cet aveugle qui apparaît dans le film de façon inattendue est, comme il l'indique, une anacoluthe : «rien dans la séquence ne semblait l'annoncer, [...] il surgit d'un coup en coupant» (Derrida, 2000, p. 79). L'image de l'aveugle effectue une rupture à l'intérieur de la construction syntaxique qu'est le film, construction qui dès lors ne devient compréhensible que par l'effort qui consiste à remplir le blanc, à suppléer au manque : « un fil secret vient toujours faufiler ou recoudre, disons dans l'inconscient, l'apparence de ce que la perception reçoit, accueille, laisse venir» (Derrida, 2000, p. 80). L'anacoluthe est césure, blessure: césure comme celle qui a lieu durant le montage d'un film, alors que certaines scènes tournées seront néanmoins jetées, le fil du tournage brisé et puis remodelé ; blessure comme celle qui a lieu lors d'une transplantation et qu'entre la coupure et la suture vient s'installer un corps étranger. (Corps étranger comme les mots de Derrida dans l'univers de mon grand-père - rien de plus éloigné, en apparence, que ces deux êtres, pôles néanmoins de mon imaginaire.) Imprévu, survenu à l'improviste, l'aveugle apparaît (Derrida affirme d'ailleurs ne pas l'avoir vu durant le tournage); et ainsi, il fait événement. Toutefois, cet événement qu'il est, l'aveugle, il ne le verra jamais (puisqu'il ne verra jamais le film). "Un peu comme moi, en somme, écrit Derrida, et comme l'Acteur!» (Derrida, 2000, p. 80) qu'il devient lui-même, à travers ce film où il joue son propre rôle, affirmant que même s'il voit à l'infini le film dont il est le sujet et l'objet, il ne le verra jamais. "À moins que [s]on témoignage, à moins que [s]on savoir allégué » sur le film «n'ajoute un surcroît de cécité. Qui serait aussi un surcroît de vérité quant au divorce » (Derrida, 2000, p. 80) entre l'Acteur et lui, entre le film et sa personne filmée. Si l'acte de témoigner a à voir avec le voir et le savoir, l'Aveugle de Tolède, qui sert de pôle à la réflexion de Derrida sur le film, vient signifier qu'il n'y a pas de «point de vue absolu» (Derrida, 2000, p. 80-81) - ni sur le film, ni sur lui, Derrida - qu'ils échappent toujours au regard, comme tout ce dont on voudrait un jour témoigner.

La mort de mon grand-père, si elle était prévisible étant donné son âge, est néanmoins arrivée à l'improviste. On ne s'y attendait pas, pas à ce moment-là, pas de cette façon-là. Et on ne s'y attend peut-être jamais vraiment, l'attendant mais sans y croire, gardant en soi l'espoir fou qu'elle n'arrivera pas. Cette mort est donc arrivée, comme le plan de l'aveugle de Tolède, en faisant rupture et événement. Comme Derrida lors du tournage du film, «je me serai laissé[e] surprendre » et prendre par une sorte d'hypnose: "Jamais je n'ai consenti à ce point », écrit ce dernier. «Jamais, comme en connaissance de cause, je n'ai ainsi agi en aveugle, les yeux fermés sur un ordre qui me dictait: Ầ ce point, à cette date, tu dois renoncer à garder, et à te garder, et à te regarder» (Derrida, 2000, p. 73). C'est à travers cette passivité hypnotisée, qui donne toutefois lieu à une action, à un jeu, que Derrida devient l'Acteur: "Que je reste étranger ("ailleurs", autre) même au regard de ma "vérité", voilà l'expérience dont je n'arriverais pas à parler mais qui me paraît devoir être au moins évoquée » (Derrida, 2000, p. 74). Ce divorce entre l'Acteur et lui avait commencé bien avant le film et avait proliféré durant toute sa vie, et c'est peut-être là la réussite du film que d'avoir reproduit, par le divorce entre l'Acteur et lui, les divorces multiples entre lui et lui ayant lieu dans sa vie ailleurs que dans le film (Derrida, 2000, p. 75). D'ailleurs, Derrida, dit le titre. Et c'est une étrangeté analogue à celle-ci que je vais tenter d'explorer aujourd'hui. Mon grand-père sera l'aveugle de Tolède et, moi, je serai comme Derrida devant le film.

Mon expérience du film: elle tend à se rassembler en ce point, désormais.

Maintenant, et pour toujours, tout s'organise, comme dans la physiologie 
de l'œil, à partir de ce qui fut, et qui reste, un point aveugle. Autour de lui, depuis sa place, mon point de vue se voit ainsi assigné. (Derrida, 2000,

\section{p. 77)}

"L'aveugle est assis, contemplez-le, arrêtez l'image. » Telle est l'injonction du philosophe, et telle est l'allégorie autour de laquelle je vais, à mon tour, tisser, tourner des mots qui formeront un voile, un filtre à travers lequel voir mon grand-père. Comme Derrida, je tracerai les contours de cet événement par le biais d'un abécédaire, nécessitant cette grammaire, le mouvement arbitraire et ritualisé de cette syntaxe (il y a peut-être quelque chose des rituels à la Sophie Calle ici, comme celui de la lettre exposé dans L'Obéissance), pour arriver à nommer ce qui m'aveugle; abécédaire qui aura pour but non pas d'offrir un lexique explicatif, un véritable dictionnaire, mais de figurer, par son semblant d'ordre, le désordre lui-même, de révéler des « rapports orageux avec la vérité» (Derrida, 2000, p. 122), c'est-à-dire avec ce que je vois. Un alphabet qui représentera, comme pour Derrida, une «rançon versée pour sauver l'aveugle de Tolède» (Derrida, 2000, p. 85).
A Disons donc que j'ai déjà commencé, en bonne et due forme, avec Ta lettre A, et que je le suivrai jusqu'au bout, tentant par cet alphabet de faire un lexique des mots entourant la mort de mon grandpère, tournant autour de cette mort comme d'un point aveugle, cette partie de l'œil qui ne voit pas mais autour de laquelle la vue s'organise. A pour aveugle, donc, mais aussi pour Alzheimer, ce dont on a dit que mon grand-père souffrait à la fin de sa vie, autre point aveugle, celui-ci relié à la mémoire. A aussi pour Aricept, un nouveau médicament qu'on lui donnait expérimentalement (et bien inutilement) pour tenter de stopper la maladie.

Comment s'écriraient des mémoires d'aveugle, demande Derrida? Comme des mémoires d'Alzheimer, des mémoires de l'oubli. Sa fille, ma mère, avait acheté à mon grand-père un cahier qui devait servir à le préserver contre l'oubli de sa propre vie, un cahier tissé de questions à lui poser, de blancs à remplir avec lui. "Où êtes-vous né ? Comment s'appellent vos parents, vos grands-parents, vos arrière-grands-parents? Quel est votre souvenir le plus ancien?» Un cahier comme le squelette d'une vie, une série de souvenirs amputés. Mais ce remplissage de blancs, était-ce pour nous plutôt que pour lui, pour nous protéger peut-être de ce qui apparaissait, à travers mon grand-père, comme la "condition amnésique de la mémoire » (Derrida, 2000, p. 93), de toute mémoire, y compris de la nôtre? Les jeunes opposent souvent leur mémoire à celle des vieux, oubliant qu'ils ont, eux aussi, des mémoires d'aveugle, qu'autant ils se souviennent, autant ils oublient. Comme si leur regard pouvait tout saisir alors que celui du vieillard ne saisissait les choses qu'à demi.

Pendant qu'elle faisait le film, Safaa Fathy a accusé Derrida d'être aveugle. «Ce fut son mot», écrit-il. "Elle m'a traité d'aveugle, elle a répété que je ne pouvais pas voir le film, et que toutes mes incompréhensions, mes impatiences, mes coups de colère, mes crises de nerfs tenaient au fait que je ne voyais rien, que je ne voyais pas, de l'autre côté, de son point de vue à elle, la vérité du film qui se préparait» (Derrida, 2000, p. 86). Fathy avait raison, admet Derrida, car aujourd'hui encore, il

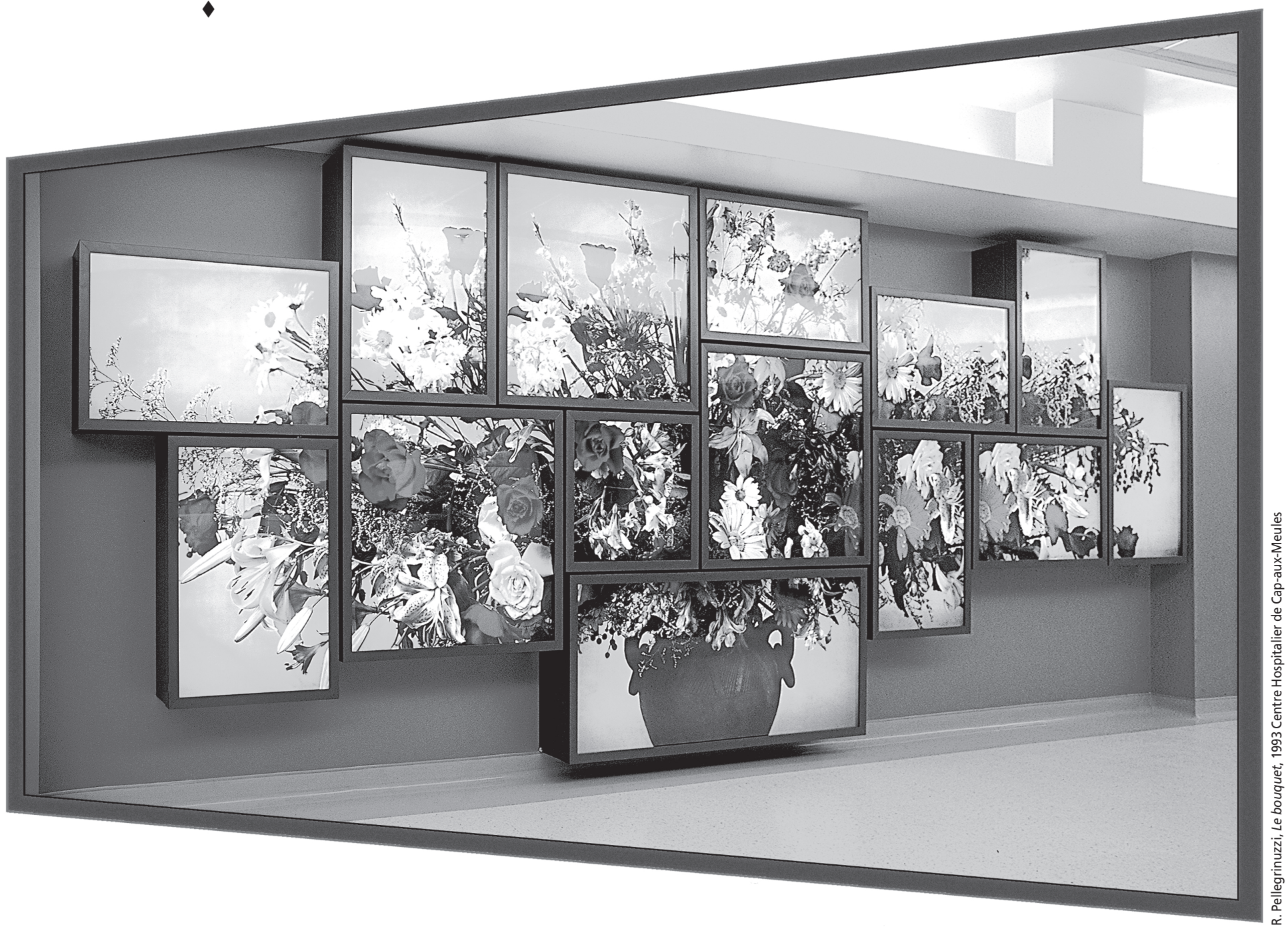


ne peut pas voir le film. Toutefois, elle non plus, selon lui, ne voyait rien, elle non plus ne pouvait pas le voir, comme s'ils souffraient tous les deux de cécité, d'une sorte de myopie qui les empêchait de discerner l'objet qui se dessinait par et devant eux.

Mon grand-père aurait été en droit de nous traiter d'aveugles. C'est un terme qu'il n'a jamais utilisé et pourtant, nous l'étions, nous l'avons été. Jusqu'à la fin, mon grandpère est resté porteur de revendications, d'une certaine colère, d'une amertume et d'une révolte quant à la vie qu'il avait et celle qu'il avait eue. J'essayais maladroitement de l'apaiser, ne me rendant pas compte que c'était plutôt moi que je calmais, frustrée de l'entendre parler ainsi de sa vie, prise par mon propre malaise comme si en se plaignant c'était moi qu'il dénigrait. Oui, il aurait pu m'accuser d'être aveugle, car si je voyais quelqu'un, c'était moi plutôt que lui. «Une mémoire apaisée n'a plus aucune chance, écrit Derrida, il ne lui reste plus qu'à s'endormir »(Derrida, 2000 , p. 93). De fait, si on refuse souvent d'entendre la colère des vieux, si on refuse de la voir, est-ce parce qu'elle figure ce qui reste en eux de vie - car tant qu'elle est là, tout n'est pas fini, la mort n'y est pas?

Cette vie qui s'achève mais qui ne finit pas, cette mort qui se présente mais qui n'arrive pas, c'est un savoir qu'on ne voit pas, le point aveugle d'où naît le point de vue. «Et comment pouvoir rester invisible à soi-même en tant que voyant, tout en ayant le savoir absolu de cette limite, de cette bordure ou de ce cerne entourant le voir? ", demande Derrida. "Comment s'approprier le savoir absolu d'une tache aveugle» (Derrida, 2000, p. 86-87) sinon en tant que témoin? Et est-ce que je n'écris pas cet abécédaire ainsi, pour tourner des lettres sur l'aveugle que mon grand-père représente afin de cerner celle qu'à travers lui je suis devenue, ne pouvant tout voir, ayant enfin appris que le regard n'est pas forcément la vue?

IR (T) Au cours des der13, 1, 19. nières semaines de sa vie, mon grand-père est devenu véritablement malade. Il a présenté des symptômes lisibles de maladie et a pu être diagnostiqué. La lettre B pour la bactérie qui s'est infiltrée par le biais de son pied pour venir miner ses forces, ce qui lui restait de santé. Bactérie non pas mangeuse mais habiteuse de chair, mettant en abyme la colonisation qui était déjà bien en place par le biais du CHSLD, ce que le ministère de la Santé du Québec appelle un Centre hospitalier pour soins de longue durée, vers lequel mon grand-père avait été démobilisé. "Avec vous... pour la vie», dit le slogan des CHSLD. Les CHSLD sont des mouroirs légitimés, des volets fermés sur les dernières années. D pour le deuil qu'un CHSLD fait vivre avant même que la vie ne soit terminée.

C'est dans cette chambre du CHSLD que mon grand-père est disparu d'une mort en apparence calme, sereine, mort que nous avons tenté d'accompagner, de cerner, d'entourer de nos corps, sachant qu'elle arrivait mais n'y croyant pas tout à fait, essayant naïvement de la préparer. Face à ce départ qui se préparait, j'ai parfois eu envie de le quitter en premier, de fuir pour ne pas voir son corps décharné, son souffle coupé, pour ne pas avoir à le voir me quitter. Mais à la fin, c'est lui qui m'a précédée, expirant durant les quelques secondes où nous l'avions laissé. Je l'ai vu vivant, et puis je l'ai vu décédé. Je n'ai pas vu le moment de sa mort, je n'ai pas pu le capturer. Point aveugle, il m'a aveuglé. Mais c'est là aussi, dans cet instant où il m'a échappé, que se trouve ce que mon grand-père m'a montré : cette incapacité de voir la mort et donc l'impossibilité d'en témoigner. Mais ce faisant, il m'a aussi montré qu'être témoin, c'est nécessairement être aveuglé. «Un témoin, en tant que tel, est toujours aveugle», écrit Derrida dans Mémoires d'aveugle; son regard repose dans ce qu'il ne voit pas, dans ce qu'il ne saisit pas, dans ce qu'il ne sera jamais capable de révéler. La main de mon grand-père dans la mienne, les soubresauts de ses membres pendant la lutte: jamais son corps n'a été à la fois si près et si loin de moi, jamais je n'ai autant cherché à saisir quelque chose qui néanmoins ne pouvait qu'éternellement m'échapper. Derrida écrit au sujet du film de Fathy: «je garde surtout la mémoire endeuillée de ce qu'on ne verra ni n'entendra peut-être jamais, et qui fut pourtant “tourné"» (Derrida, 2000, p. 110). De même, je souffre de ce statut qui consiste à voir et à ne pouvoir jamais réellement témoigner, sinon imparfaitement comme je le fais sans doute maintenant, en écrivant sur lui.

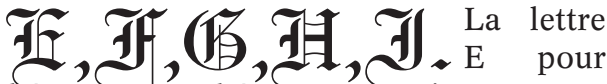
l'écriture, pour l'écrivain qu'était son propre père, médecin de campagne et auteur de romans de la terre, et pour ce que lui, mon grand-père, n'a jamais pratiqué une fois marié, laissant à son épouse le soin de parler sur papier, comme je le fais ici. Un peu comme Derrida cherchant à sauver l'aveugle de Tolède, préservant non seulement la vision de celui qui ne voit pas, mais la sienne comme point de vue aveuglé. L'aveugle de Tolède que Derrida n'a pas vu durant le tournage et dont il n'a constaté la présence qu'une fois le film monté, l'aveugle lui «ne verra jamais le film. Un peu comme moi, en somme», écrit Derrida, et comme l'Acteur. Mais il ne pourra même pas témoigner, comme je le fais ici, de notre aveuglement commun »(Derrida, 2000, p. 80).
L'aveuglement de l'aveugle qui ne verra jamais le film n'est pas le même que celui de Derrida, Acteur, qui ne pourra jamais, même s'il le voit des milliers de fois, voir le film dont il est le sujet. À moins qu'en fait il ne soit le seul à le voir «de l'autre côté», son témoignage révélant, en l'aveuglant, la vérité. Écrivant sur l'aveugle, Derrida ne témoigne donc pas pour lui, en tâchant de remplacer sa vue, mais témoigne plutôt de l'aveuglement, d'une impossible accession à la vérité, au point de vue absolu. Et c'est un tracé analogue que je dois suivre ici, trouvant dans le point aveugle qu'est la mort de mon grand-père ce qui donne un sens à ma vue. D'avoir été présente et de ne pas avoir vu est bien ce qui m'affilie à lui. F, donc, pour cette syntaxe qu'est la filiation, organisation de gens comme autant de mots à la tête desquels il se tiendrait, chef de file d'une série qui commencerait par le G de Gérard, son prénom.

(À la lettre G, j'avais aussi indiqué le mot grenouillère, drôle de mot qui ferait sans doute sourire. Mais quand on sait que la grenouillère est cette camisole de force qui a pour but d'enfermer les vieux dans une couche souillée, en les empêchant de pouvoir la retirer... Le port de la grenouillère, qui a été forcé, a précipité le décès de mon grand-père, sa disparition dans un au-delà psychologique provoquée par l'humiliation - et voilà bien la lettre $\mathrm{H}$ qui apparaît - et par la déshumanisation. Savoir médical et information sur la santé ? I pour infirmières et infirmiers qui se font les gardes des prisons et des dossiers.)

f) que ce grand-père souhaitait autour de lui comme autant d'espoir, de beauté. Et par homonymie, le mot jeûne par lequel le désir de mort a commencé à se manifester. Puis les kilos qui s'effaçaient progressivement de sa chair jusqu'à ce qu'il ne reste presque plus rien, que des os menaçant de percer la peau appuyée sur des coussins. Il était devenu plus menu que moi, celle qui pourtant avait toujours été plus petite que lui, celle dont il s'était occupé quand elle était bébé, qui avait souvent dormi sur lui. L pour le lit dans lequel il a beaucoup dormi, épuisé par une existence dont il refusait néanmoins de se libérer. Un sommeil qui, lui aussi, a pris les traits d'une préparation à la mort. Il s'endormait devant moi, m'indiquant ainsi qu'il était temps de partir, me préparant à son effacement, comme s'il avait décidé qu'il me fallait voir ses yeux se fermer.

La lettre M est chargée: M comment le nier, et pour la mort qui est maintenant arrivée. $\mathrm{M}$ pour la morphine que j'ai accepté de lui voir donnée durant les dernières heures pour calmer l'angoisse, les soubresauts du corps, les élans de survie. 
Cette morphine qui hante désormais mes nuits comme un résidu de culpabilité, une douce euthanasie... Voilà mon image de l'aveugle: ce plan de mon grand-père recroquevillé sur son lit, à demi parti et pourtant toujours présent, une image qui a arrêté le temps. Il avait à ce moment-là retrouvé une position d'enfant, et nous caressions ses membres, nous l'enveloppions, hypnotisés, le souffle coupé suivant le rythme rompu du sien, dans l'attente de l'ultime captation: le dernier respir, l'image de la fin. $\mathrm{N}$ enfin pour la nourriture que durant ces instants j'ai ponctuellement ingurgitée, mangeant pour le préserver, pour le garder. Une fois la mort accomplie, l'accompagnement terminé, je me suis tournée vers lui que j'avais inconsciemment incorporé, le re-gardant encore et encore pour ne le libérer, peutêtre, que maintenant.

\section{$\mathbb{B}, \mathfrak{A}, \mathbb{Q}, \mathfrak{R}, \mathbb{S}, \mathbb{C}$.}

Ce grand-père, j'y arrive, était pour moi une langue, un nom, une origine, en somme, un père. Pour moi l'Ontarienne, comme il aimait bien m'appeler, il était, lui, l'incarnation d'un Québec idyllique, de racines fantasmées par le biais de ce lieu à demi fictif dont il provenait et qui s'appelle SaintHilaire, du nom de cet évêque de Poitiers moyenâgeux dont la réflexion sur la théologie trinitaire précéda celle de SaintAugustin. (Ou serait-ce le Saint-Hilaire d'Arles, celui-là adversaire d'Augustin, ou encore le Saint-Hilaire de Sardaigne qui aura été pape... Je ne savais pas qu'il y avait plus d'un Saint-Hilaire. Je me dis aujourd'hui que ça ferait sourire mon grand-père.) S'il n'était pas très pratiquant, sinon pour faire plaisir à son épouse (et peut-être pour la faire taire), il était néanmoins croyant. Mais son dieu à lui accueillait sûrement les plaisirs de la vie, de la chair, petit grandpère rigolo, lubrique, créatif... La lettre $S$ pour les safaris qu'il me faisait imaginer les matins où, toute petite, je le rejoignais dans son lit pour l'entendre raconter comment les hélicoptères atterrissaient derrière chez lui pour permettre à des chasseurs d'entreprendre une immense chasse aux lions. Les récits qu'il me racontait, je peux encore les faire tourner dans mon esprit, et c'est ça aussi qu'il m'aura légué: le souvenir de l'enfant que j'étais qui, sûrement mieux que la femme que je suis aujourd'hui, était capable de rejoindre le rêve qu'il incarnait. Lui disparu, est-ce que je me retrouve face à ma vie comme Derrida devant le film de Safaa Fathy: un "Spectateur ahuri, un Rêveur qui n'arrive pas à crever la toile du Rêve, un Spectateur-Acteur parfois incapable de traverser l'écran pour rentrer dans le film, pour le rejoindre » (Derrida, 2000, p. 82) ? Et est-ce là le chemin que je dois faire à l'aveuglette, cherchant dans le noir, à tâtons, la brèche qui se trouve peut-être au bas de l'écran et que je pourrais ouvrir comme une enveloppe, m'y glissant comme un mot, une lettre? La lettre $\mathrm{T}$, par exemple : il y en a trois dans le surnom qu'il me donnait quand je lui demandais s'il savait qui j'étais. Il répondait, sourire en coin, «Martinette».

H Je me dirige doucement vers la fin, 12. glissant mes pas vers les dernières Tettres. Peu de mots commencent en U, mais bon nombre d'entre eux commencent par « un », et je volerai donc à Derrida celui qu'il a lui-même choisi, un comme dans Dieu un, unique et universel, un aussi comme dans unité et unicité, et vous me voyez venir avec mes gros souliers, pour dire comment ce grand-père l'a signifié. Il était l'unique, le seul, le mien. Et il y a sûrement quelque chose d'étrange dans le fait que je suis en train d'en parler, moi, universitaire presque chevronnée. On pourrait sûrement m'accuser d'un attachement attardé (et impudiquement affiché). Mais, comme l'écrit Derrida, «ce qui m'est le plus propre, indéracinablement propre, ne m'appartiendra jamais » (Derrida, 2000, p. 115), et l'écriture n'a peut-être pour but que cela, d'arriver à cet ailleurs, ce point aveugle où il se trouve et à partir duquel je tente désormais de me trouver.

H Je ne me pencherai pas plus avant 14. sur la lettre V que je n'ai cessé d'employer - dans voir, savoir, vieillissement -, sinon pour en venir finalement à la question du voile, le voile qui recouvrait progressivement la cornée de mon grandpère et que des verres ne parvenaient pas à soulever. Voile aussi qui se manifestait sous la forme de larmes se logeant en apparence sans raison au creux de ses yeux, et qu'il avait fini par laisser couler. Sa peau même était devenue un voile, une surface parcheminée. Durant son enfance, mon grandpère a souffert d'un eczéma lancinant, son épiderme déchiré par des plaies s'ouvrant sur des fenêtres de douleur. À la fin de sa vie, au cours des dernières années, miroir des toutes premières, il a été atteint d'un pemphigus, une affection rare, un trouble auto-immunitaire qui se manifeste par une érosion de la peau. La carapace s'amincit, la surface s'effrite, devient transparente, le sang s'épanche, l'intérieur se livre à l'extérieur. Le vieillissement est la progression d'un effacement, la disparition des muscles, de la chair, faisant place à l'apparition des os, comme si le corps, s'inversant comme un gant, se dé-voilant, devenait lui-même une sorte de voile, rideau se levant sur la mort.

Nous avions choisi d'accompagner mon grand-père, mais il se devait de partir en solitaire. "Bénédiction de qui part sans laisser d'adresse », écrit Derrida dans Un ver à soie. «Ne plus s'être ni s'avoir, voilà la vérité sans vérité qui me cherche au bout du monde. Faire son deuil de la vérité [...] mais (ou donc) sans porter ni faire porter le deuil à personne »(Derrida, 2000, p. 43). Savoir chaotique, regard partiel sur mon grand-père, je vois cet abécédaire comme autant de fils qui se tissent autour de lui en un linceul renfermant à jamais l'impression de ses traits. Il disparaît, il se retire, vérité sans vérité dont il ne me demande pas de faire le deuil. Il m'apprend à prendre le voile, à le garder, à savoir que le papillon frétille à l'intérieur du cocon bien avant de s'en dégager. Le voile: pour Derrida, c'est la figure du soi (dans Un ver à soie). «En finir avec le voile, c'est en finir avec soi », écrit-il (Derrida, 2000, p. 30), et cela est impossible. Le soi ne se dévoile pas; il demeure toujours mouvement de voile. Ce qui lui fait dire que de rompre avec l'Un, avec le fantasme de ce quelqu'un que l'on serait, avec notre visage imprimé sur un suaire, rompre avec l'Un «sans laisser de trace [...] voilà le suicide absolu et le sens premier qu'il peut y avoir à laisser vivre l'autre, le laisser être, sans même escompter le moindre bénéfice de ce retrait du voile ou du linceul» (Derrida, 2000, p. 43).

Hes dernières phabet ne sont pas des lettres françaises. Derrida en fait les frais lui aussi quand il se retrouve devant elles. Pour les inscrire, je pourrais choisir des noms de lieux (anglais, allemands) ou encore de médicaments: mon grand-père aimait les voyages, et il était pharmacien avant d'être malade. Mais je ne garderai ici, pour dernière lettre, que le Y, d'abord pour nommer «Yvette», la femme qu'il aimait, et puis, en trichant un peu, pour en revenir à l'œil, c'est-à-dire aux yeux. «Faut-il des yeux à la naissance d'un regard ? », demande Derrida (Derrida, 2000, p. 125). Peut-être qu'il n'en faut pas, ou que le regard n'est jamais tout à fait celui des yeux qui regardent. Peut-être faut-il apprendre à regarder tout en y renonçant, en renonçant à se garder et à se regarder en se gardant.

Écrire, donc, pour sauver l'aveugle de Tolède. Écrire pour restituer la vue de l'aveugle, pour habiter ses yeux. Mais surtout: écrire à l'aveugle, voire écrire en double-aveugle comme je l'ai fait ici, ne sachant pas qui, de lui ou de moi, était le témoin. Car au bout du compte, au bout de ces lettres, c'est bien de lui et de moi qu'il s'agit, du fantasme du moi laissé pour compte pour lui, l'aveugle de Saint-Hilaire, d'ailleurs, mon grand-père.

\section{Bibliographie}

DERRIDA, Jacques (2000). D'ailleurs, Paris, Galilée. 